\author{
FELIX GROLLMANN \\ Ludwig-Maximilians-Universität München \\ E-Mail: felix.grollmann@gmx.de
}

\title{
GESTALTUNGSSPIELRÄUME STÄDTISCHER REGULIERUNG VON GEWÄSSERN VOR UND NACH DEN BAYERISCHEN WASSERGESETZEN VON 1852
}

Hinsichtlich der Gewässer im urbanen Raum liegt eine potentiell konfliktträchtige Situation vor. Diese Spannung erwächst aus dichten Siedlungsverhältnissen mit einem differenzierten Bedarf an Nutzungen einerseits und der beschränkten Verfügbarkeit von Wasser andererseits: in der Stadt besteht gegenüber dem, flachen Land' generell ein größerer Bedarf für Wasserrecht. Wechselwirkungen von Mensch und Wasser werden durch Recht aufgegriffen und gleichzeitig konstituiert. Im Folgenden sollen sowohl die normative Einrahmung der Stadt als auch die Festsetzung allgemeiner Vorschriften sowie die (verschiedentlich stark regelgeleitete) Behandlung von Einzelfällen durch die Stadt betrachtet werden - andere,weiche' Phänomene, die für die Realisierung bzw. Etablierung von Herrschaftsansprüchen ebenfalls von großer Bedeutung sind, wie symbolische Kommunikation und Rituale, werden dagegen außer Acht gelassen ${ }^{1}$. Der zeitliche Schwerpunkt dieser Abhandlung liegt im 19. Jahrhundert. Damals wurden im Königreich Bayern drei Wassergesetze erlassen, die zum ersten Mal in der

1 Einen breit gespannten rechtsgeschichtlichen Überblick enthält H. Seiler, Die Gewässerbenutzungen und ihre Rechtsgrundlagen im Verlauf der Geschichte des Wasserrechts. Ein vergleichender Überblick, (1976), passim. Vgl. auch R. Heydenreuther, Die Isar in der Rechtsgeschichte. Anmerkungen zur Entwicklung des bayerischen Wasserrechts, in: Die Isar: ein Lebenslauf. Ausstellung im Münchner Stadtmuseum vom 5. Mai bis 25. September 1983, hg. v. M.-L. Plessen, (1983), S. 52-63. 
bayerischen Geschichte eine systematische und überschaubare Gesetzeslage zum Wasserrecht schufen ${ }^{2}$. Besondere Aufmerksamkeit hieraus gilt dem Gesetz über die Benützung des Wassers vom 28. Mai 1852² da dieses (auch im Verhältnis zu den beiden anderen Gesetzen) grundlegend für das bayerische Wasserrecht war ${ }^{4}$. Die zweite Hälfte des 19. Jahrhunderts bildet eine Zäsur in der Geschichte des Wasserrechts, da auch in anderen Territorien erstmalig ,große' Gesetze auf diesem Gebiet entstanden. Räumlich steht die Haupt- und Residenzstadt München im Mittelpunkt. Dies ist, anders als die zeitliche Konzentration, begründungsbedürftig. Trotz gewisser Lockerungen gegenüber den Zuständen ${ }^{5}$ zu Beginn des 19. Jahrhunderts, als bezeichnenderweise sogar das Stadtwappen abgeschafft worden war, konnte sich München 1852 nur eingeschränkt selbst verwalten ${ }^{6}$. Um ausgeprägte städtische Herrschaft unter die Lupe zu nehmen, müsste der Fokus auf eine Reichsstadt wie Nürnberg vor dem Ende ihrer eigenstaatlichen Zeit gelegt werden. Im Folgenden ist aber gerade ein Blick darauf zu werfen, welche Befugnisse und Gestaltungsspielräume einer Stadt verblieben, welche allein schon wegen der ansässigen Residenz unter besonderer monarchischer Aufmerksamkeit stand ${ }^{7}$.

${ }^{2}$ Es handelt sich um das Wasserbenützungsgesetz, das Be- und Entwässerungsgesetz sowie das Uferschutzgesetz.

3 Gesetz über die Benützung des Wassers (vom 28. Mai 1852), in: Gesetzblatt für das Königreich Baiern, (1851/1852), Sp. 489-542 (von nun an: WBG).

${ }^{4}$ Vgl. H. Reuß, Die bayerischen Wassergesetze vom 28. Mai 1852: mit Ergänzungsgesetzen, Vollzugserlassen, Motiven, oberstrichterlichen Erkenntnissen und Erläuterungen, (Die bayerischen Kulturgesetze nebst Vollzugsvorschriften 3, 1890), S. XII.

${ }^{5}$ Vgl. R. Bauer, Stadt und Stadtverfassung im Umbruch. Niedergang, Ende und Neubegründung kommunaler Eigenständigkeit 1767 bis 1818, in: Geschichte der Stadt München, hg. v. dems., (1992), S. $268 \mathrm{f}$.

${ }^{6}$ R. Zerback, Unter der Kuratel des Staates - Die Stadt zwischen dem Gemeineedikt von 1818 und der Gemeindeordnung von 1869, in: Geschichte der Stadt München, hg. v. R. Bauer, (1992), S. 244-273.

${ }^{7}$ Diese Fragestellung berührt sich mit den jüngeren rechtshistorischen Forschungen zur ,Regulierten Selbstregulierung. Überlegungen zu Kommunen im 19. Jahrhundert bei P. Collin, Einleitung: „Gesellschaftliche Selbstregulierung “ und „Regulierte Selbstregulierung“ - ertragreiche Analysekategorien für eine (rechts-)historische Perspektive?, in: Selbstregulierung im 19. Jahrhundert. Zwischen Autonomie und staatlichen Steuerungsansprüchen, hg. v. dems. u. a., (Schriften zur europäischen Rechtsgeschichte 259, Moderne Regulierungsregime 1, 2011), S. 10; ders., Kommunalrecht unter Regulierungsdruck in der Weimarer Zeit, in: Regulierte Selbstregulierung im frühen Interventions- und Sozialstaat, hg. v. dems. u. a., (Schriften zur europäischen Rechtsgeschichte 270, Moderne Regulierungsregime 2, 2012), S. 146 f. 


\section{VOR- UND ENTSTEHUNGSGESCHICHTE DES WASSERBENÜTZUNGSGESETZES}

Das Wasserrecht hatte lange Zeit den Ruf, ein besonders schwieriges Rechtsgebiet zu sein ${ }^{8}$. Dies korreliert mit einer verwickelten Dogmengeschichte. Erstmalig wird der Ausdruck, Wasserrecht' in einem Traktat eines Hof-Kammerrats im Jahr 1570 verwendet. ${ }^{9}$ Diese begriffliche Verdichtung in einem gelehrten Werk ist bezeichnend. Von herrschaftlicher Seite sind Wasserrechte, wobei die Plural-Form bewusst eingesetzt wird, in Privilegien eingeräumt oder in Erlassen in sachlichem Zusammenhang mit anderen Rechtsgebieten, wie dem Baurecht, Strafrecht oder dem allgemeinen Stadtrecht ${ }^{10}$, geregelt worden - ausdifferenzierte Normwerke speziell zum Wasserrecht sind rechtsgeschichtlich vor dem 19. Jahrhundert indes vernachlässigbar. ${ }^{11}$ Im Hochmittelalter wurde die Verleihung von Wasserrechten als Regalrecht vereinnahmt, im Zuge der Ausbildung der Landesherrschaften geriet sie dann zunehmend in die Verfügungsgewalt der territorialen Machthaber ${ }^{12}$. Wasserrechte entwickelten sich über lange Zeit hinweg induktiv und abhängig von den jeweiligen Umständen. Die Inhaberschaft einzelner Wasserrechte wurde auch durch die Gesetzgebungen des 19. und 20. Jahrhunderts nicht abrupt beendet. Bis in die Gegenwart spielt die Frage nach der Fortgeltung von Altrechten eine große Rolle für die Gewässerbenutzungspraxis. ${ }^{13}$ So gibt es in München nach wie vor An-

${ }^{8}$ Vgl. M. R. Franz, Rechtliche Bestimmungen zum Wasserbau im Herzog- und Kurfürstentum Bayern, in: Altbayerische Flußlandschaften an Donau, Lech, Isar und Inn: handgezeichnete Karten des 16. bis 18. Jahrhunderts aus dem Bayerischen Hauptstaatsarchiv, (1998), S. 290.

9 Dazu Seiler, Gewässerbenutzungen, S. $1 \mathrm{f}$.

${ }^{10}$ Beispiele bei Heydenreuther, Die Isar in der Rechtsgeschichte, S. $55 \mathrm{f}$.

11 Überblick: Seiler, Gewässerbenutzungen, S. 30-38.

12 Hierzu knapp Heydenreuther, Die Isar in der Rechtsgeschichte, S. 52 f.

13 So regelt Art. 75 des Bayerischen Wassergesetzes vom 25. Februar 2010 (Bayerisches Gesetzes- und Verordnungsblatt 2010, S. 66-98) das Fortbestehen von Altrechten unter Verweisung auf das Bayerische Wassergesetz vom 23. März 1907 (Gesetz- und Verordnungsblatt für das Königreich Bayern, 1907, S. 157-212). Dort wurden in Art. 207 Altrechte bestätigt. Unter dem Regime des Wasserbenützungsgesetzes sind Altrechte ebenfalls als fortgeltend angesehen worden. Vgl. J. Pözl, Die bayerischen Wassergesetze vom 28. Mai 1852. Mit einem Anhange, die Ordnung der Schiff- und Floßfahrt auf den bayerischen Flüssen, Seen und Kanälen betreffend, (1862), zu Art. 104 WBG, S. 241: „Nur die abweichenden älteren Bestimmungen sind aufgehoben, also die Rechtsnormen, - nicht auch die Rechte der 
wesen, die mit sog. Ewigsteften verbunden sind, womit Wasserrechte aus der Zeit vor 1791 bezeichnet werden ${ }^{14}$. Eine weitere Konstanz, welche im Rahmen der Gesetzgebungen des 19. Jahrhunderts zu berücksichtigen war, lag in der Dichotomie von öffentlichen und privaten Gewässern - diese resultierte aus gelehrten Rechtsvorstellungen und hatte Konsequenzen für die Möglichkeit, den Zugang und die Benutzung der Gewässer zu steuern bzw. von Zahlungen oder sonstigen Leistungen abhängig zu machen ${ }^{15}$.

Entsprechend diesen generellen Beobachtungen richteten sich auch für Bayern und insbesondere für die Stadt München Wasserrechte einerseits nach einzelnen Privilegien (zum Beispiel wenn die Stadt im Spätmittelalter die herzogliche Zustimmung für die Betreibung von Mühlen benötigte ${ }^{16}$ ) und andererseits nach verstreuten, an größere Adressatenkreise ergangenen Vorschriften ${ }^{17}$. So ergaben sich etwa aus den Landrecht, Policey-GerichtsMalefitz- vnd andere Ordnungen von 1616 Regelungen für Mühlwerk und

Einzelnen und Korporationen, welche unter der Herrschaft des früheren Rechtes in giltiger Weise erworben worden sind. Sie bestehen auch unter der Herrschaft des neuen Gesetzes fort; nur versteht es sich von selbst, daß über deren Ausübung die jetzigen Vorschriften entscheiden." Es sei nur beispielhaft auf den schon lange schwelenden und kürzlich wieder einmal aktualisierten Konflikt der Stadtwerke München GmbH, einer 100\&-igen Tochter der Landeshauptstadt München, mit dem Landkreis Miesbach um die Beziehung von Trinkwasser aus dem Mangfalltal verwiesen; hierbei ist der Inhalt von Altrechten aus dem 19. Jahrhundert maßgeblich. Vgl. M. Köpf, Wem gehört das Wasser?, in: Süddeutsche Zeitung, (3. August 2018): URL: https://www.sueddeutsche.de/ muenchen/mangfalltal-wem-gehoert-das-wasser-1.4080518 (aufgerufen 10.09.2018). Zur stadtpolitischen Entstehung der Münchner Trinkwasserversorgung aus dem Mangfalltal: P. Münch, Stadthygiene im 19. und 20. Jahrhundert: die Wasserversorgung, Abwasser- und Abfallbeseitigung unter besonderer Berücksichtigung Münchens, (Schriftenreihe der Historischen Kommission bei der Bayerischen Akademie der Wissenschaften 49, 1993), S. 186-193.

14 Hierzu Münch, Stadthygiene, S. 124.

15 Vgl. aus germanistischer Sicht die Darstellung der Entwicklung dieses Prinzips bei H. Geffcken, Zur Geschichte des deutschen Wasserrechts, in: Zeitschrift der Savigny-Stiftung. Germanistische Abteilung, 21 (1900), S. 173-217. Zur Bedeutung dieser Zweiteilung für das WBG: M. Seydel, Bayerisches Staatsrecht, 5 (1891), S. $409 f$.

16 Vgl. C. Rädlinger, Geschichte der Münchner Stadtbäche, (2004), S. 21.

17 Vgl. zur Einschätzung der bayerischen Vorgeschichte des WBG aus der Sichtweise des 19. Jahrhunderts: Seydel, Bayerisches Staatsrecht, 5, S. 407, wonach die „Bestimmungen des bayerischen Landrechtes von 1756 [...] ziemlich spärlich [sind]“ und sich im Übrigen nur „Bruchstücke öffentlichrechtlicher Vorschriften“ finden ließen. Wasserrechtliche Bestimmungen im Münchner Stadtrecht, in dem Landrecht Kaiser Ludwigs des Bayern und Vorschriften des 15. und 16. Jahrhundert werden überblickt bei: Franz, Rechtliche Bestimmungen, S. $291 \mathrm{f}$. 
Fischerei, also zwei wirtschaftlich relevanten Benutzungsarten ${ }^{18}$. Und im Codex Maximilianeus Bavaricus civilis (1756) waren Wasserbenutzungen (etwa durch Schöpfen) als Felddienstbarkeit eingeordnet worden ${ }^{19}$. Aber auch die Stadt selber setzte Recht mit Bezug auf die Gewässer fest. In der vom Rat und Bürgermeister erlassenen Bauordnung von 1613 war etwa die jährliche Pflicht zur Räumung der Flüsse geregelt ${ }^{20}$. Schließlich gab es auch Fälle gemeinsamer Normsetzung, etwa in Gestalt der Mühlordnung von 1487, die von Herzog Albrecht IV. (reg. 1465-1508) und dem Rat der Stadt München ausging ${ }^{21}$.

Diese nicht leicht überschaubare Rechtslage vereinfachte sich 1852 schlagartig ${ }^{22}$. Vor der Entstehung des WBG stand der Entwurf eines Wiesenkulturgesetzes, den die Staatsregierung in den Landtag von 1846 einbrachte. ${ }^{23}$ Primärer Regelungsgegenstand war die Erleichterung der Durchführung von Be- und Entwässerungsunternehmen durch Enteignungen und andere Zwangsmaßnahmen. Freilich setzte dieses Gesetz Eigentum und Benutzungsrechte an Gewässern nur vage voraus, was von Seiten des Landtags als unvollständig angesehen wurde. Dies war der entscheidende Anstoß, warum die Staatsregierung dem Landtag von 1851/1852 einen Gesetzesentwurf betreffend der Benützung des Wassers vorlegte. An der endgültigen Fassung war der Landtag, der einen besonderen Ausschuss mit 15 Mitgliedern gebildet hatte, maßgeblich beteiligt. Als ein bezeichnendes Beispiel für einen solchen Einfluss kann die Streichung des Art. 3 des Entwurfs genannt werden ${ }^{24}$. Dieser sah vor, dass die Staatsregierung durch Beschluss

18 Landrecht, Policey- Gerichts- Malefitz-vnd andere Ordnungen. Der Fürstenthumben Obern vnd Nidern Bayrn, (1616), lib. IV, tit. VIII und IX.

19 Vgl. Das bayerische Landrecht (Codex Maximilianeus Bavaricus civilis) vom Jahre 1756 in seiner heutigen Geltung, hg. v. M. Danzer, (1894), 2, VIII. Kapitel, § 12, S. $111 \mathrm{f}$.

20 Rädlinger, Geschichte, S. 72.

21 W. Kohl, Recht und Geschichte der alten Münchner Mühlen, (Miscellanea Bavarica Monacensia 15. Neue Schriftenreihe des Stadtarchivs München 32, 1969), S. 90 f. Zur allgemeinen Rechtsentwicklung: H. Lück, Mühle, Mühlenrecht, in: Handwörterbuch zur Deutschen Rechtsgeschichte, 3 (2016), Sp. 447-448, wonach Mühlrecht in der Frühen Neuzeit nur gelegentlich verschriftlicht wurde.

22 Die Uneindeutigkeit der Rechtslage vor 1852 bezüglich der Finanzierung von Wasserbauten betont Franz, Rechtliche Bestimmungen, S. 294.

23 Zum Folgenden Pözl, Die bayerischen Wassergesetze, S. 7f.; Seydel, Bayerisches Staatsrecht, 5, S. 407f.

24 Dazu Pözl, Die bayerischen Wassergesetze, zu Art. 2 WBG, S. $28 f$. 
einen Fluss für schiff- und flößbar und damit für öffentlich erklären konnte. Dies hätte den Fluss durch bloße Willensäußerung zu Staatsgut gemacht ${ }^{25}$. Das erschien dem Landtag sowohl im Regelungskontext unstimmig als auch mit dem Prinzip der Rechtssicherheit für unvereinbar - diese und andere Einschränkungen seiner Regierungsgewalt akzeptierte der König, der das Gesetz im Landtagsabschied vom 28. Mai 1852 sanktionierte.

Was sind nun die Motive der Entstehung des Wasserbenützungsgesetzes gewesen, welches - wie bereits erwähnt - eine Zäsur für die Gesetzgebungsgeschichte bedeutete? Dies ist umso erklärungsbedürftiger, als Bayern mit seinen Gesetzen von 1852 nicht allein dastand. So gab es neben den bayerischen Gesetzen u. a. für Braunschweig seit 1851, für Baden seit 1876, für Hessen-Darmstadt seit 1887 und für Elsass-Lothringen seit 1891 systematische und wenigstens vom Anspruch her umfassende Wasserrechtsgesetze. Mehrere Gründe für dieses Phänomen sind erkennbar. Die Gesetzgebung lässt sich jedenfalls nicht allein als Verrechtlichungsvorgang verstehen. Sind doch gerade im bürgerlichen Zeitalter des 19. Jahrhunderts Tendenzen zur Deregulierung zum Tragen gekommen, wenn man etwa an die Aufhebung der Zünfte und die Einführung der allgemeinen Gewerbefreiheit denkt ${ }^{26}$. In Bayern wurde die volle Gewerbefreiheit zwar erst mit der Gewerbeordnung vom 30. Januar 1868 etabliert; dieser Reform waren aber immerhin kleinere Liberalisierungen vorangegangen ${ }^{27}$. Hinzukommt zumindest noch der Vorbildcharakter von abschließenden und einheitlichen Kodifikationen seit dem ausgehenden 18. Jahrhundert, wie etwa das Allgemeine Landrecht für die preußischen Staaten (1794) oder der Code civil (1804) ${ }^{28}$. Solche Ge-

25 Zur rechtswissenschaftlichen Diskussion im 19. Jahrhundert über die Bedeutung von Staatsgut Seiler, Gewässerbenutzungen, S. 215-217.

26 C. Opitz, Art. Verrechtlichung, in: Historisches Lexikon der Schweiz, URL: http://www. hls-dhs-dss.ch/textes/d/D25615.php, aufgerufen 10.09.2018).

27 R. Gömmel, Gewerbe, Handel und Verkehr, in: Handbuch der bayerischen Geschichte, 4: Das neue Bayern, von 1800 bis zur Gegenwart, 2: Die innere und kulturelle Entwicklung, (2007), S. 231f.

28 Der kodifikatorische Charakter des WBG wird zeitgenössisch prägnant beschrieben bei Reuß, Die bayerischen Wassergesetze, S. XI f.: „Seit dem 7. Oktober 1852 regelt das Wasserbenützungsgesetz alle wasserrechtlichen Verhältnisse an erster Stelle, im ganzen Lande und für alle Einwohner. Entgegenstehendes Recht muß als abgeschafft gelten. Es gilt sonach in ganz Bayern, gleichmäßig im Hauptlande wie in der Rheinpfalz, ferner [...]. Herkommen und Gewohnheit sind nur da als Rechtsquellen anzusehen, wo diese das Wasserbenützungsgesetz ausdrücklich zuläßt, und insofern sie es zuläßt: vgl. Art. 104. Neubildung von Gewohnheitsrecht dürfte mit dem Gesetze unvereinbar sein“. 
setzeswerke standen als Modell für das systematische Erfassen eines großen Sachkomplexes in einem Gesetz zum Zwecke der Normenklarheit und Rechtssicherheit zur Verfügung. Und schließlich sind ökonomische Aspekte als Faktor nicht zu vernachlässigen. Die in der 2. Hälfte des 19. Jahrhunderts entstandenen Gesetze waren auch eine Reaktion auf die gesteigerten Benutzungsbedürfnisse durch die Industrialisierung, wie es für das Herzogtum Braunschweig detailliert nachgezeichnet worden ist ${ }^{29}$. Diese Beobachtungen lassen sich nicht ohne weiteres auf das in der Mitte des 19. Jahrhunderts noch stark agrarisch geprägte Königreich Bayern übertragen. Dass die strukturellen Veränderungen durch die Industrialisierung aber jedenfalls innerhalb des zeitgenössischen Wahrnehmungshorizonts der Staatsführung lag, zeigt sich darin, dass König Maximilian II. (reg. 1848-1864) in seinem Antrittsjahr einen Unterstützungsfonds für industrielle Zwecke schuf ${ }^{30}$. Schwer hiervon als Entwicklungsfaktor zu trennen ist die gesteigerte Verkehrsbedeutung der Flüsse im 19. Jahrhundert ${ }^{31}$.

In dieses Bedingungsgefüge lässt sich die Entstehung des WBG einordnen. So monierte Joseph Pözl, Professor der Rechte an der Universität München, in seinem Kommentar zu den bayerischen Wassergesetzen im Jahr 1862, dass es vor 1852 an einer ,einheitlichen und gleichförmigen Regel für die Gewässer überhaupt“ gefehlt habe und ,die einzeln vorhandenen

29 C. Behrens, Die Wassergesetzgebung im Herzogtum Braunschweig nach Bauernbefreiung und industrieller Revolution. Zur Genese des Wasserrechts im bürgerlichen Rechtsstaat, (Rechtsgeschichtliche Studien 30, 2009), S. 51-89. In Bezug auf das WBG wird bei Reuß, Die Bayerischen Wassergesetze, S. XIII, ausgeführt, dass die Industrie darin kein Hauptgedanke sei. Damit ist freilich nicht ausgeschlossen, dass für das WBG die intensivierte Wassernutzung infolge der Industrialisierung ein wichtiger Entstehungsfaktor war. Und damit ist auch nicht gesagt, dass die sich nach 1852 weiterhin in Veränderung begriffenen ökonomischen Verhältnisse (neben weiteren Umständen) nicht zum Bayerischen Wassergesetz von 1907 führten. Zur wirtschaftlichen Entwicklung als einer Entstehungsursache der Reform von 1907 C. Meisner, Das in Bayern geltende Nachbarrecht: mit Berücksichtigung des Berg- und Wasserrechts, (1901 und $\left.{ }^{2} 1910\right)$, S. 395 ( $\left.{ }^{2} 1910\right)$ : „So sehr die Wassergesetzgebung von 1852 auf der Höhe ihrer Zeit gestanden hatte, so erwies sie sich doch infolge der Umgestaltung des wirtschaftlichen Lebens und der hierdurch bedingten außerordentlichen Ansprüche an die Wasserversorgung immer mehr als unzulänglich, um einen billigen Ausgleich der Interessenkonflikte unter den veränderten Umstände zu schaffen“.

30 Hierzu und allgemein zur Industrialisierung Bayerns (publiziert am 22.01.2007): H. Braun, Industrialisierung, in: Historisches Lexikon Bayerns, URL: http://www.historisches-lexikon-bayerns.de/Lexikon/Industrialisierung, aufgerufen 8.10.2018).

${ }^{31}$ Heydenreuther, Die Isar in der Rechtsgeschichte, S. 52f. 
positiven Normen unvollständig und den thatsächlichen und wirtschaftlichen Bedürfnissen der Gegenwart" nicht angemessen gewesen wären ${ }^{32}$. Das WBG beseitigte, so Pözl, diese Defizite überwiegend, wenn auch nicht vollständig, da das Wasserrecht auf drei Gesetze verteilt und einige Bereiche, wie die Verhältnisse der Seen, nicht berücksichtigt wurden ${ }^{33}$. Erst mit dem Wassergesetz für das Königreich Bayern von 1907 gab es ein zentrales Gesetzeswerk.

\section{GEMEINDEVERFASSUNGSRECHT UND NORMATIVE BEFUGNISSE DER STADT MÜNCHEN}

Zur Orientierung können nur einige Eckpunkte herausgestellt werden. Wie schon angesprochen, waren die autonomen Elemente der Stadtverfassung um 1800 erheblich zurückgedrängt worden ${ }^{34}$. Am 31. Dezember 1802 war München und anderen Städten die Gerichts- und Polizeihoheit entzogen worden ${ }^{35}$. Mit dem Edikt über das Gemeinde-Wesen ${ }^{36}$ von 1808 büßten die Gemeinden „ihre kommunale Eigenständigkeit [...] voll und ganz ${ }^{{ }^{* 37}}$ ein.

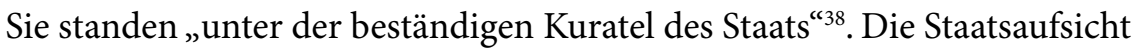
wurde erst in der Gemeindeordnung von 1869 in Fach- (bei Aufgaben im übertragenen Wirkungskreis) und Rechtsaufsicht (bei Aufgaben im eigenen Wirkungskreis) unterteilt ${ }^{39}$. Für das Entstehungsjahr des WBG spielt die Qualifizierung von städtischen Aufgaben als solche des eigenen bzw. übertragenen Wirkungskreises insoweit keine Rolle. Daher wird dieser

32 Pözl, Die bayerischen Wassergesetze, S. 4.

33 Ebenda, S. 5.

34 Einen leicht zugänglichen Überblick über die Entwicklung des bayerischen Gemeindeverfassungsrechts liefert (publiziert am 11.05.2006): E. Mages, Art. Gemeindeverfassung (19./20. Jahrhundert), in: Historisches Lexikon Bayerns, URL: https://www. historisches-lexikon-bayerns.de/Lexikon/Gemeindeverfassung_(19./20._Jahrhundert); aufgerufen 5.10.2018).

35 Dazu Zerback, Unter der Kuratel des Staates, S. 268.

${ }^{36}$ Edikt über das Gemeinde-Wesen (vom 24. September 1808), in: Königlich-baierisches Regierungsblatt, (1808), Sp. 2405-2431 (von nun an: Gemeindeedikt (1808)).

37 F.-L. Kneymeyer, Die bayerischen Gemeindeordnungen 1808-1945, (Schriften zur öffentlichen Verwaltung 41, 1994), S. 1.

38 Gemeindeedikt (1808), $\$ 8$.

39 Dazu Mages, Art. Gemeindeverfassung. 
Aspekt im vorliegenden Beitrag vernachlässigt. Die städtischen Aufgaben im Bereich der Gewässer regeln mehrere Vorschriften ${ }^{40}$. Ab diesem Jahr gab es in München eine Polizeidirektion, die staatliche Aufgaben zusammen mit dem Magistrat ausübte ${ }^{41}$. Normiert war dies in der Instruktion der Polizei-Direktionen in den Städten vom 24. September $1808^{42}$. Insbesondere die Erhaltung von Wasserleitungen und Brunnen war gemäß $\$ 70$ eine Aufgabe der Polizei-Direktion, die diese zusammen mit der Baukommission zu erfüllen hatte.

Mit dem sog. Gemeindeedikt von 1818 wurde die kommunale Eigenständigkeit in Ansätzen erneuert ${ }^{43}$. Das Gemeinderecht von 1808 hatte sich als „nicht voll realisierbar“ erwiesen ${ }^{44}$. Freilich dauerte die staatliche Kuratel fort. Von Selbstverwaltung lasse sich im strengen Sinne erst ab 1869 sprechen $^{45}$. Bezeichnenderweise wurden die Gemeinden durch den Gesetzgeber teilweise Minderjährigen gleichgestellt ${ }^{46}$. Der Münchner Magistrat unterstand der Aufsicht der Kreisregierung ${ }^{47}$. Von dieser sollte er Befehle empfangen ${ }^{48}$. Zu den (überschaubaren) Kompetenzen der Städte, die sich insbesondere aus dem Amtseid erschließen lassen ${ }^{49}$, gehörten

40 Vgl. ebenda, $\$ \$ 15,351), 48$.

41 Dazu W. Volkert, Innere Verwaltung, in: Handbuch der bayerischen Ämter, Gemeinden und Gerichte 1799-1980, hg. v. dems., (1983), S. 91.

42 Instruktion der Polizei-Direktionen in den Städten (vom 24. September 1808), in: Königlich-Baierisches Regierungsblatt, (1808), Sp. 2509-2532. Vgl. Gemeindeedikt (1808), $\$ 108$.

${ }^{43}$ Königliche Verordnung: die künftige Verfassung und Verwaltung der Gemeinden im Königreiche betreffend (vom 17. Mai 1818), in: WBG, (1818), Sp. 49-96 (von nun an: Gemeindeedikt (1818)).

44 E. Weis, Die Begründung des modernen bayerischen Staates unter König Max I. (1799-1825), in: Handbuch der bayerischen Geschichte, 4: Das neue Bayern, von 1800 bis zur Gegenwart, 1: Staat und Politik, (2003), S. 107.

45 S. Fisch, Stadtplanung im 19. Jahrhundert. Das Beispiel München bis zur Ära Theodor Fischer, (1988), S. 36.

46 Gemeindeedikt (1818), \$21: „Sie [sc. die Gemeinden] stehen unter der besonderen Curatel und Aufsicht des Staates, und genießen die Vorrechte der Minderjährigen“.

$47 \mathrm{Zu}$ den genannten Aspekten Volkert, Innere Verwaltung, S. $88 \mathrm{f}$.

48 Vgl. Gemeindeedikt (1818), § 122: „In den Städten der ersten Classe [sc. wozu auch München gehörte] ist der Magistrat - als eine selbstständige Behörde - der Kreis Regierung unmittelbar untergeordnet. Er berichtet hiernach unmittelbar an die Regierung, und empfängt von dieser in gleicher Art die ihm zugehenden Befehle [...]“.

49 Nach Gemeindeedikt (1818), $\$ 45$ wird die gemeindliche Verwaltung in den 
die Vermögensverwaltung und auch die Wasserversorgung, während die Aufrechterhaltung der öffentlichen Sicherheit und Ordnung prinzipiell der Polizeidirektion oblag; ausgenommen davon war nur die Lebensmittelpoli$z^{5}{ }^{50}$. Die Zuständigkeiten im Bereich der Wasserverwaltung sind in einigen Vorschriften enthalten, die teilweise dem Edikt von 1808 ähneln ${ }^{51}$.

Für die Haupt- und Residenzstadt galt unter der Geltung des Edikts von 1818 ein besonderes Gemeindeverfassungsrecht ${ }^{52}$. Mit der königlichen Verordnung vom 19. September 1818 wurde das Verhältnis von Magistrat und Polizeidirektion unter grundsätzlicher Fortgeltung der zuvor erwähnten Instruktion aus dem Jahr 1808 reguliert ${ }^{53}$. Nach $\$ 94$ der Verordnung waren sowohl Magistrat als auch Polizei-Direktion bloß vollziehende und verwaltende Behörden, welche Verordnungen nicht ändern konnten, sondern an diese nur erinnern sollten. Zuständig war der Magistrat nach $₫$ 51 für die Vorsorge für Wasserschäden, nach $\$ 64$ grundsätzlich für die Herstellung und Unterhaltung von Brunnen und Wasserleitungen sowie (mit der Möglichkeit der "geeigneten Einwirkung" durch die Bau-Kommission) nach $\$ 66$ für den Brücken- und Wasserbau. In den Aufgabenkreis der Polizei-Direktion fielen dagegen nach $\$ 51$ die Vorkehrungen für die Sicherheit bei tatsächlich realisierten Wassergefahren ${ }^{54}$. Insgesamt lässt sich

Städten und größeren Märkten durch den Magistrat, den Gemeinde-Ausschuss sowie in größeren Städten den District-Vorsteher besorgt und vollzogen. Die Aufgabenverteilung zwischen diesen Einrichtungen ergibt sich aus Gemeindeedikt (1818), §\$ 46-92.

50 Zerback, Unter der Kuratel des Staates, S. 275.

51 Vgl. Gemeindeedikt (1818), $₫ 65$ : „Er [sc. der Magistrat] sorgt für die Herstellung der Gemeinde-Wege, Brücken und Stege, der Brunnen und Wasserleitungen, insoweit diese aus Gemeinde-Mitteln bestritten werden“ mit Gemeindeedikt (1808), $\$ \$ 15,351$ ). Im älteren Edikt wurden Wasserleitungen nicht ausdrücklich erwähnt. Im Enteignungsgesetz von 1837 wurde geregelt, dass die Herstellung öffentlicher Wasserleitungen ein möglicher Zweck von Enteignungen ist. Hierzu Seydel, Bayerisches Staatsrecht, 5, S. 411.

52 An maßgeblicher Stelle wurde dem Münchner Magistrat in $\$ 67$ Gemeindeedikt (1818) eine Aufgabe nicht zugewiesen: „Dem Magistrate ist in allen Städten und Märkten, mit Ausnahme unserer Haupt- und Residenzstadt München, als Regierungs-Beamten, die gesammte Local-Polizey übertragen“.

53 Königliche Verordnung: das Verhältnis zwischen der Polizey-Direction und dem Magistrate der Haupt- und Residenz-Stadt München betreffend (vom 15. September 1818), in: WBG, (1818), Sp. 573-616. Vgl. \$ 70 Gemeindeedikt (1818).

54 Diese Zuständigkeiten bzw. Aufgabenverteilung lässt schon $₫ 27$ Gemeindeedikt (1818) erkennen: „Zur Herstellung und Ausbesserung der nöthigen Gemeinde-Gebäude, Brunnen, Wege, Brücken, der Ufer- und Wasser-Bauten etc.; in so fern sie der Gemeinde 
festhalten, dass die Zuständigkeiten der Stadt München für den Umgang mit Gewässern seit der Rechtslage von 1808 modifiziert und teilweise gestärkt worden sind.

Erweitert und insoweit erheblich genauer gefasst wurden die Aufgaben der Haupt- und Residenzstadt München dann 1852 mit dem WBG. Ausgangspunkt für die Zuständigkeit der Stadt war Art. 92, der auf die allgemeinen Kompetenzvorschriften verwies und festlegte, dass, soweit das Gesetz von Verwaltungsbehörde sprach, jeweils die Distrikts-Verwaltungsbehörde zuständig war, womit die Stadt München als sog. unmittelbare Stadt in erster Instanz tätig wurde. Andere Entscheidungsträger waren die Staatsregierung, die etwa nach Art. 1 Regelungen für öffentliche Gewässer erlassen konnte, oder die Gerichte, die beispielsweise über die öffentliche Eigenschaft von stehenden Gewässern gemäß Art. 7 im Falle eines Streites $\mathrm{zu}$ entscheiden hatten ${ }^{55}$.

Die wichtigsten, ausdrücklichen ${ }^{56}$ Kompetenzen der Stadt als Verwaltungsbehörde in der I. Abteilung, welche die öffentlichen Flüsse betrifft (Art. 1-32), waren: bei Errichtung oder Abänderung von Dämmen oder ähnlichen Anlagen, die auf den Wasserlauf oder die Höhe des Wasserstands Einfluss haben können, innerhalb der Überschwemmungsgebiete von öffentlichen Flüssen benötigte der Betreiber eine sog. Erlaubnis ${ }^{57}$, die das Maß der Benützung festlegte; dasselbe galt für sämtliche Vorrichtungen, die den Lauf des Wassers störten (Art. 10-12) (58 $^{58}$ In den Motiven wurde das Recht der Verwaltung zum unmittelbaren Einschreiten hervorgehoben ${ }^{59}$.

obliegen, zur Handhabung der öffentlichen Sicherheit, soweit nicht auf andere Art dafür hinreichend gesorgt ist, sind die Gemeinden Frohnen oder Gemeinde-Dienste zu leisten schuldig“. Nach $\$ 91$ 1) Gemeindeedikt (1818) hatten die Distrikts-Vorsteher (soweit solche bestellt waren) die Aufsicht über Brücken, Wege, Stege, Brunnen und Wasserleitungen.

55 Meinungsverschiedenheiten der Gerichte bei der Annahme ihrer Zuständigkeiten werden angesprochen bei Reuß, Die bayerischen Wassergesetze, S. XIII.

56 Nach Art. 9 waren bestimmte Benutzungen geringerer Intensität von öffentlichen Gewässern allgemein erlaubt, solange keine entgegenstehenden polizeilichen Vorschriften bestanden. Hierunter dürften auch Erlasse der Gemeinden gefallen sein. Vgl. zu Art. 9 WBG: Pözl, Die bayerischen Wassergesetze, S. 48.

$57 \mathrm{Zu}$ dessen Rechtsnatur und insbesondere und seiner privatrechtlichen Bedeutung Seiler, Gewässerbenutzungen, S. 291-293.

58 Gemäß Art. 13 könnten solche Erlaubnisse nicht ohne weiteres zurückgenommen werden.

59 Abgedruckt bei Reuß, Die Bayerischen Wassergesetze, S. 19: zu Art. 10 WBG. 
Art. 15, der Abführen von Steinen, Sand, Schlamm, Erde und Pflanzen aus dem Flussbett von einer Erlaubnis ${ }^{60}$ abhängig machte, bestätigte in Absatz 3 das Fortbestehen von Altrechten: hier kam zugunsten der Stadt München der am 7. November 1724 bestätigte Burgfriedensbrief von 1460 zum Tragen, aus welchem sich ergab, dass die Stadt befugt war, aus dem Flussbett der innerhalb des Burgfriedens gelegenen Isar Steine etc. abzuführen bzw. Dritten gegen Geldleistung zu überlassen ${ }^{61}$. Der städtische Gestaltungsspielraum ergab sich also aus einer anderen Rechtsgrundlage, die aber vom WBG anerkannt wurde. Mit dem Gesetz vom 22. Februar 1855 wurden diese Privilegien erneut bestätigt ${ }^{62}$. In den Regelungen des WBG zu den öffentlichen Gewässern finden sich noch weitere Aufgaben der Verwaltungsbehörde ${ }^{63}$.

In der II. Abteilung des Gesetzes (Art. 33-65), welche die Privatflüsse zum Gegenstand hat, wurde in den ersten Vorschriften vor allem der Eigentumsinhalt festgesetzt ${ }^{64}$. In diesem Regelungskontext wurde die Stadt bei Konflikten infolge eines veränderten Flussbetts tätig, indem sie auf Verlangen der Beteiligten nach Art. 41 eine Frist für die Wiederherstellung rechtmäßiger Zustände zu setzen hatte ${ }^{65}$. Dass Privatflüsse durch Behörden

60 Zur Rechtsnatur der Erlaubnis Seiler, Gewässerbenutzungen, S. $230 f$.

${ }^{61}$ Vgl. Pözl, Die bayerischen Wassergesetze, S. 67: zu Art. 15 WBG.

${ }^{62}$ Vgl. $\$ 1$ Gesetz vom 22. Februar 1855, die Statuar- und Gewohnheits-Rechte der $k$. Haupt- und Residenzstadt München betreffend (abgedruckt bei Reuß, Die Bayerischen Wassergesetze, S. 353): „Die Statuar- und Gewohnheitsrechte der Stadt München kommen in dem ganzen Umfange der Burgfriedensgrenze, soweit sich dieselbe dermalen erstreckt oder künftig erstrecken wird, zur Anwendung".

63 Nach Art. 19 hatte die Stadt die Uferlinie festzusetzen, womit das Privateigentum der Anlieger (Art. 18) begrenzt wurde. Diese Maßnahme war Verwaltungssache. So Seydel, Bayerisches Staatsrecht, 5, S. 412. Eine weitere, für den Verkehr flussaufwärts wichtige Befugnis, war die Regelung des Leinpfads durch die Verwaltungsbehörde, Art. 20 WBG. Die Beseitigung von Anschütten war der Verwaltungsbehörde zu bestimmten Zwecken gestattet, Art. 24 WBG.

${ }^{64}$ Zur Normstruktur der II. Abteilung: Seiler, Gewässerbenutzungen, S. 217. Die eigentumsrechtlichen Implikationen der bayerischen Wassergesetze (vornehmlich des WBG) werden systematisch dargestellt bei Meisner, Das in Bayern geltende Nachbarrecht, S. 182-261 (1901).

65 Seit der Geltung des Verwaltungsgerichtshofgesetzes von 1878 waren Streitigkeiten im Zusammenhang mit diesem Konflikt eindeutig eine Verwaltungsrechtssache. Vgl. Reuß, Die Bayerischen Wassergesetze, S. 70: zu Art. 41. Im Ergebnis auch Seydel, Bayerisches Staatsrecht, 5, S. 424, Anm. 6. 
unter öffentlich-rechtlichen Gesichtspunkten reguliert wurden, ist in den Entstehungsmaterialien damit begründet worden, dass (auch) diese Flüsse enge Beziehungen zur öffentlichen Ordnung hätten ${ }^{66}$. In der allgemein gehaltenen Vorschrift des Art. 52 wurden die Behörden mit der Aufsicht über die Privatflüsse beauftragt und ermächtigt, bei Vorliegen eines allgemeinen Interesses polizeiliche Anordnungen zu treffen ${ }^{67}$. $\mathrm{Zu}$ dieser allgemeinen Regelung traten noch zahlreiche weitere Befugnisnormen hinzu. Die Aufgaben der Verwaltungsbehörden waren im Vergleich zur I. Abteilung des Gesetzes konkreter geregelt ${ }^{68}$. Gegenüber den Gerichten war ihr Einfluss dennoch wesentlich geringer ${ }^{69}$. Nur ausnahmsweise war die Kreisregierung erstinstanzlich zuständig (etwa im Falle von Art. 62 f.). Weitere Aufgaben der Verwaltungsbehörden ergaben sich auch aus dem Abschnitt zu Triftgewässern (Art. 71) sowie zu Triebwerken und anderen Stauvorrichtungen (Art. 73, 78 ff.). Bezüglich der Zwangsrechte zur Beförderung der Wasserbenützung (Art. 86-91) waren Gerichte zuständig. Einzelne Normverstöße im Bereich der Zuständigkeit der Verwaltungsbehörden waren mit Geldstrafen bewehrt (Art. 96-98); Polizeistrafen konnte die Stadt als Verwaltungsbe-

${ }^{66}$ Wiedergegeben bei Pözl, Die bayerischen Wassergesetze, S. 140: zu Art. 50 WBG.

67 Als Grund für die behördliche Aufsicht wurde in den Motiven die „Fürsorge gegen die der Gesundheit schädlichen Einflüsse" (abgedruckt bei Reuß, Die Bayerischen Wassergesetze, S. 83: zu Art. 52 WBG) genannt, die auch bei Privatgewässern zu beachten sei. Unter den Anordnungen wurden später sowohl allgemeine als auch einzelne verstanden: Seydel, Bayerisches Staatsrecht, 5, S. 427 m. w. N.

68 Insbesondere war die Stadt zuständig für die Anordnung der Uferreinigung und Ausräumung des Flussbetts (Art. 49-51). Benutzungen von geringerer Intensität, wie Baden, waren in Parallelität zu Art. 9 allgemein erlaubt; die Verwaltungsbehörde durfte hier polizeiliche Anordnungen treffen (Art. 53). Zum Verhältnis von Art. 9 und 53 vgl. Pözl, Die bayerischen Wassergesetze, S. 147 f.: zu Art. 53 WBG. Abweichungen von Einschränkungen der Benützung des vorbeifließenden Wassers durch die Ufereigentümer konnten von der Verwaltungsbehörde zugelassen werden (Art. 54). Die Ableitung von Wasser für die Betreibung von Anlagen konnte durch die Verwaltungsbehörde beschränkt werden (Art. 56). Das Erfordernis einer Bewilligung der Verwaltungsbehörde für die Benützung der Gewässer, durch welche die Gewässereigenschaften negativ verändert werden (z. B. zum Betrieb von chemischen Fabriken), war nach Art. 58 vorgeschrieben. Hierzu Seiler, Gewässerbenutzungen, S. 249. Bei vermindertem Wasserstand konnte die Verwaltungsbehörde die Verteilung des Wassers festlegen (Art. 60). Im Zusammenhang mit Triebwerken gab es Anzeigepflichten zugunsten der Verwaltungsbehörde, damit diese ihre Aufgaben effektiv erfüllen konnte (Art. 61). Bei nutzloser Verschwendung von Wasser sollte sie ebenfalls tätig werden (Art. 64).

69 So Seiler, Gewässerbenutzungen, S. 273. 
hörde ebenfalls festsetzen (Art. 100). Die Differenz zwischen Geld- und Polizeistrafen bestand darin, dass bei ersteren schon der Verstoß gegen das Gesetz sanktioniert, während bei Polizeistrafen das Zuwiderhandeln gegen behördliche Anordnungen maßgeblich war ${ }^{70}$. Aus Art. 101 ergab sich, dass der Magistrat der Stadt für die Verfolgung von Polizeiübertretungen zuständig war ${ }^{71}$.

Es lässt sich festhalten, dass München als Verwaltungsbehörde in beträchtlichem Umfang Aufsichts-, Entscheidungs- und Handlungsrechte besaß. Dies galt gleichermaßen für alle unmittelbaren Städte, sodass sich aus dem Gesetz selbst keine besonderen Rechte für die Haupt- und Residenzstadt ergaben. Privilegien beruhten auf Rechten außerhalb des WBG, die von demselben aber anerkannt sein mussten. Nach 1852 wurden wohl aus Gründen der Rechtssicherheit die Privilegien der Stadt München erneut bekräftigt. Im Verhältnis zur skizzierten Reform von 1818 lässt sich für das Inkrafttreten des WBG eher von einer Ergänzung als von einer Verdrängung sprechen. Denn die Verordnung und das Gemeindeedikt regelten (in teilweiser Kontinuität zur Rechtslage von 1808) Kompetenzen für gemeinwohlbezogenes Handeln durch Bau und Unterhaltung von Wasserleitungen, Brunnen und Brücken, während das Gesetz von 1852 stärker die repressive Seite des Wasserrechts im Verhältnis von Behörde und Individuum betraf, indem das Handeln von Privaten zumindest beaufsichtigt, nicht selten auch von einer behördlichen Erlaubnis abhängig gemacht wurde; Wasserbenutzung konnte in Strafen (bei Verstößen gegen gesetzliche Bestimmungen oder behördliche Anordnungen) resultieren.

\section{STÄDTISCHE GESTALTUNGSSPIELRÄUME NACH 1852}

Nach heutigem Gemeinderecht und allgemeinem Verwaltungsverfahrensrecht wird das Verwaltungshandeln von Kommunen insbesondere durch zwei Vorgaben vor übermäßiger Beschränkung durch die Staatsgewalt geschützt, wodurch sich zugleich behördliche Gestaltungsspielräume ergeben: die Aufsichtsbehörden des Freistaats Bayern dürfen Kommunen,

70 Reuß, Die Bayerischen Wassergesetze, S. 153: zur VII. Abteilung. Dazu auch Seydel, Bayerisches Staatsrecht, 5, S. 439.

71 Vgl. Pözl, Die bayerischen Wassergesetze, S. 232 f.: zu Art. 101 WBG. 
soweit diese Aufgaben im eigenen Wirkungskreis erfüllen, nur hinsichtlich der Rechtmäßigkeit ihres Handelns, aber nicht hinsichtlich der Zweckmäßigkeit kontrollieren ${ }^{72}$. Gerichte können das kommunale (wie jegliches behördliches) Handeln, soweit es auf einer ermessenseinräumenden sog. Kann-Regelung beruht, nur unter Einschränkungen überprüfen ${ }^{73}$.

Im Untersuchungszeitraum um 1850 ist Ermessen zwar als Rechtsbegriff schon etabliert gewesen ${ }^{74}$, es markierte aber den Bereich insgesamt justizfreier politischer Entscheidung, betraf also nicht die Kontrolldichte zuständiger Gerichte ${ }^{75}$.Vielmehr war der Rechtsschutz gegen die öffentliche Gewalt nach dem Zusammenbruch des Alten Reichs insbesondere infolge der nun durchgesetzten Lehre von der landesherrlichen Souveränität und der Verengung des Privatrechts auf die Summe der gleichgeordneten Rechte und Gegenrechte der Untertanen verkürzt worden ${ }^{76}$. Aufgrund der strengen staatlichen Kuratel (zwischen 1808 und 1869) über die bayerischen Gemeinden konnte deren Handeln staatlicherseits ohnehin vollständig überprüft werden; diese Kontrolle konnte jederzeit mittels Rekurs durch die Betroffenen eingeleitet werden. Vor 1852 war ein solches Einschreiten zwar ebenfalls möglich, aber mangels konkreter Rechtsvorgaben für die höheren Instanzen nur ineffizient durchführbar. Hinzukam, dass zahlreiche Einwirkungen auf die Gewässer mangels typisierter Gestattungspflichten obrigkeitlich schlicht nicht wahrgenommen wurde. Die städtische Autonomie wurde 1852 erneut spürbar eingeengt.

Trotzdem verblieben auch nach 1852 noch kleine Gestaltungsspielräume. In den von mir durchgesehenen Akten aus dem Stadtarchiv München

72 Vgl. Art. 109 Abs. 1 der Gemeindeordnung für den Freistaat Bayern (in der Fassung der Bekanntmachung von 22. August 1998).

$73 \mathrm{Zu}$ den Grenzen des Ermessens Verwaltungsverfahrensgesetz, hg. v. P. Stelkens, H. J. Bonk, M. Sachs, (2018), Rn. 53-130: zu $\$ 40$.

74 Für das Tätigwerden von Behörden auf dem Gebiet des Wasserrechts wird der Ermessensbegriff zeitgenössisch etwa verwendet bei Seydel, Bayerisches Staatsrecht, 5, S. $417 \mathrm{f}$.

75 Zur Entwicklung zwischen 1806 und den 1860er Jahren U. Held-Daab, Das freie Ermessen: von den vorkonstitutionellen Wurzeln zur positivistischen Auflösung der Ermessenslehre, (Schriften zum öffentlichen Recht 706, 1996), S. 53-69.

${ }^{76}$ Hierzu Held-Daab, Das freie Ermessen, S. 54-57. Die Ursache hierfür war insbesondere die „bürokratische [...] Professionalisierung und Zentralisierung durch die spätabsolutistischen Verwaltungsreformen", da damit eine Kontrolle der Verwaltung jenseits der (zudem inzwischen unabhängigen) Justiz gewährleistet war. 
sind drei Möglichkeiten sichtbar geworden, wie auch unter dem Regelungsregime des WBG die Stadt noch relativ eigenmächtig agieren konnte.

1) Wenn die Stadt als Verwaltungsbehörde wasserrechtliche Gestattungen erließ, die keine erhebliche Drittbelastung enthielten, bestanden für die Betroffenen keine Anreize, eine Beschwerde einzulegen ${ }^{77}$. Eine Kontrolle seitens der Kreisregierung war dann aber nur noch von Amts wegen möglich. Solange die Stadt die höheren Behörden über relevante Vorgänge auf diesem Rechtsgebiet nicht informierte, konnte die Stadt hier Vorhaben und sonstige Einwirkungen genehmigen, ohne dass es zur Bestimmung von Recht- und Zweckmäßigkeit durch die Kreisregierung oder das Staatsministerium kam. Vielleicht vor diesem Hintergrund erging 1890 ein auch in München abgelegtes ministerielles Rundschreiben, dass die unteren Behörden hinsichtlich der Informationspflichten ermahnte, dass man über neue Anlagen zu wenig Kenntnis erlangen würde; damit könne die staatliche Aufsicht aber nicht effektiv ausgeübt werden ${ }^{78}$.

2) Eine weitere Möglichkeit wird in einem Vorgang aus den frühen 1920er Jahren sichtbar ${ }^{79}$. Durch die „ungeheure Geldentwertung“ wurden Zahlungen, die die Stadt aufgrund der Genehmigung der Errichtung von Wasserrädern, Schlamprädern und ähnlichen Anlagen an den Stadtbächen seit der Mitte des 19. Jahrhunderts fortlaufend einnahm, zunehmend bedeutungslos. Diese altrechtlichen Genehmigungen waren , auf Ruf und Widerruf" erteilt und an die Bedingung einer Geldzahlung geknüpft worden. Sie stützten sich in einem Fall sogar ausdrücklich auf Art. 54 des WBG und waren jedenfalls durchgehend in Beschlussform gehalten. Art. 54 setzte fest, dass jeder Ufereigentümer das an seinem Grundstück vorbeifließende Wasser genehmigungsfrei nutzen konnte, wenn dadurch fremde Grundstücke durch einen Rückstau oder dergleichen nicht gefährdet und das genutzte Wasser wieder in den Fluss eingeleitet wurden, bevor dieser das Ufer eines fremden Grundstücks berührte; die Verwaltungsbehörde konnte Abweichungen hiervon erlauben. 1920 wollte die Stadt die nicht mehr adäquat erscheinenden Zahlungen erhöhen. Das Tiefbauamt beauftragte deshalb am 13. Juli 1920 ein internes Rechtsgutachten, um zu klären, ob der Stadtrat als „Wasserbenützungsberechtigter“ im Sinne des Wassergesetzes von 1907

\footnotetext{
77 Zur Berufung an die höhere Instanz M. Seydel, Bayerisches Staatsrecht, 5, S. 438.

78 Enthalten in Stadtarchiv München, Sign. DE-1992-BUG-007.

79 Dokumentiert ist der Vorgang in Stadtarchiv München, Sign. DE-1992-BUG-571.
} 
eine rechtliche Möglichkeit habe, die Zahlungen anzupassen. Dabei ging man in einer ergänzenden Frage davon aus, dass bei einem Verkauf oder einem andersweitigen Besitzübergang am Wasserrad ohne weiteres Zutun die Wasserausnützung an sich auf den Besitznachfolger mit übergehe. Insoweit nahm das anfragende Tiefbauamt einen dinglichen Charakter der Genehmigungen an. Für die Zukunft wurden Erleichterungen prognostiziert. Denn das Bayerische Wassergesetz (von 1907) sehe allein noch eine Anzeige gemäß Art. 48 vor. Hier solle der Stadtrat in jedem Einzelfall eine passende „Gebühr“ festsetzen können. Das Gutachten vom 27. Juli 1920 kam zu dem Ergebnis, dass die vermeintlichen öffentlich-rechtlichen Erlaubnisse tatsächlich Pachtverträge waren, da die Stadt die Grundstücke in ihrem Eigentum zur Nutzung durch Wasserräder überlassen hatte. Denn Art. 54 des WBG stelle einen ganz bestimmten Rahmen für das Tätigwerden der Wasserpolizeibehörde auf. Die Zahlung von Pachtzinsen sei gerade keine „Bedingung aufgrund dieser Bestimmung“. Es ging also schon unter dem WBG eigentlich um ein Privatrechtsverhältnis zwischen der Stadt und dem Wasserradbetreiber. Einen dinglichen Charakter besaßen diese Verträge nicht. Dementsprechend könnten die Verträge unter Zustimmung beider Seiten geändert werden, um eine Erhöhung herbeizuführen. Unter Zugrundelegung der Regeln des noch jungen Bürgerlichen Gesetzbuchs von 1900 hob der Verfasser hervor, dass auf ewig geschlossene Verträge ohne Abänderungsmöglichkeit sittenwidrig und nach $\$ 138$ des Bürgerlichen Gesetzbuchs nichtig wären. Der Gutachter empfahl, dass sich die Stadt, wenn der privatrechtliche Charakter ,aus irgendwelchen Gründen“ nicht offengelegt werden sollte, auf die Widerrufbarkeit stützen und so (offenkundig einseitig) die Bedingungen ändern solle. Dies könne schon nicht unbillig sein, weil sich die „Lasten für die ordnungsgemässe Erhaltung der Stadtbäche" für die Stadtgemeinde erhöht haben. Abschließend stellte das Gutachten klar, dass für die Genehmigung neuer Wasserradanlagen ein Pachtzins verlangt werden könne, der freilich keine Gebühr im Rechtssinne sei. Das Tiefbauamt entschied sich weiterhin am öffentlich-rechtlichen Charakter festzuhalten. In einem Schreiben vom 10. September 1920 wurde befürchtet, dass die Erledigung der Angelegenheit wohl noch einige Zeit dauern würde, da „Einsprüche“ durch Beteiligte zu erwarten seien. Am 21. Februar 1921 wurde dann in einem Beschluss der Stadt der Pachtzins in den überwiegenden Fällen einseitig um das 6-fache angehoben. Als Gründe wurden die Geldentwertung sowie die erheblichen Instandhal- 
tungskosten angeführt. Will man die ältere Praxis, die immerhin für eine größere Zahl von Gestattungen zur Anwendung kam, genereller verorten, kann man sagen, dass die Verwaltung durch die Extension des WBG in Privatrechtsverhältnisse hinein gegenüber den Bürgern den Eindruck der Gesetzesgebundenheit erzeugen konnte, sodass man potentiell umständlicheren Vertragsverhandlungen aus dem Weg ging. Eine Überprüfung durch Rekurs an die höheren Behörden war angesichts des synallagmatischen und damit zumindest teilweise begünstigenden Charakters der Pachtverträge auch hier unwahrscheinlich und bis Anfang der 1920er Jahre anscheinend auch nicht vorgefallen.

3) Vor 1800 war es auch in einer landesherrlichen Stadt wie München nicht atypisch, dass die Kommune sich eigenes Recht gab, wofür als Beispiel bereits die Bauordnung von 1613 genannt wurde. Dass die Stadt aber nach 1852 noch eigene wasserpolizeiliche Vorschriften erließ, war nicht mehr selbstverständlich. Dies erklärt, warum in einem Schreiben des Staatsministerium des Innern vom 17. März 1867, das an die Kreisregierung von Oberbayern adressiert war, Argumente für die Zulässigkeit der Normgebung durch die Stadt München zusammengestellt wurden. Erstens wurden Art. 52 des WBG, welcher von polizeilichen Anordnungen sprach, und Art. 164 des Polizeistrafgesetzbuchs, welcher Übertretungen des Gesetzes sowie der aufgrund dieses Gesetzes erlassenen Verordnungen für strafbar erklärte, angeführt. Zweitens wurde auf frühere polizeiliche Bestimmungen für die Flüsse in der Stadt München, bei deren Übertretung die Gerichte Strafen verhängt hätten, verwiesen. U. a. auf dieser Rechtsgrundlage könnte die Stadt am 26. August 1864 in Reaktion auf das WBG ortspolizeiliche Vorschriften zur Beachtung der Münchner Verhältnisse erlassen haben ${ }^{80}$ - im Stadtarchiv sind allerdings bloß handschriftliche Fassungen überliefert ${ }^{81}$. Dagegen ist im Amtsblatt kein entsprechendes Normenwerk publiziert worden, obwohl jenes eigentlich das Amts- und Anzeigeblatt des Stadtmagistrats, der Polizeidirektion, der Lokalbaukommission und des Bezirksamts München war ${ }^{82}$. Die Lokalbaukommission war seit 1852 in einer „Zwitterstellung“" zwischen Magistrat und Polizeidirektion ${ }^{83}$. In demselben

\footnotetext{
${ }^{80}$ Davon geht aus Rädlinger, Geschichte, S. 91, 116.

81 Stadtarchiv München, Sign. DE-1992-BUG-007 und DE-1992-BAUA-TB-0493.

82 Etwa Münchener Amtsblatt, (1864), S. 2.

${ }^{83}$ Münch, Stadthygiene, S. 143.
} 
Jahr haben die Polizeidirektion und Lokalbaukommission Vorschriften über Abtritte erlassen, die am sachlich passenden Ort im Münchener Amtsblatt bekanntgemacht worden sind; in $\$ 14$ wurde explizit auf die Beachtung des WBG bei der Einmündung von Abtritten in fließendes Wasser hingewiesen ${ }^{84}$. Es ist schwer erklärlich, warum der Münchener Magistrat dagegen die Vorschriften vom 26. August 1864 überhaupt nicht oder an anderer Stelle hätte publik machen sollen.

Jedenfalls 1877 sind Vorschriften durch den Magistrat und die Lokalbaukommission zur Einleitung von Entwässerungen in den Schlachthauskanal ergangen, die u. a. genaue Vorgaben zu den Entwässerungsanlagen enthielten ${ }^{85}$. So legte $\$ 3$ fest, aus welchem Material die Entwässerungsleitungen konstruiert werden durften und wie die einzelnen Röhren zu verbinden waren. Im folgenden Jahr wurde im Erlass vom 7. Juni 1878 die Abwasserentsorgung in München genereller geregelt. In $₫ 1$ wurde von allen Besitzern von Anwesen, die an Straßen liegen, in welchen die Stadt Kanäle errichtet hat, die Herstellung von Entwässerungsanlagen verlangt; gemäß $\$ 3$ waren auf diese Anlagen die Vorschriften von 16. November 1877 entsprechend anwendbar. Beide Erlasse mussten durch die Regierung von Oberbayern für vollziehbar erklärt werden. Es ist aufschlussreich, dass der Erlass von 1877 in einem Entwurfsfassung noch auf Art. 52 des WBG gestützt worden war, während in der amtlichen Fassung u.a. das Polizeistrafgesetzbuch als Rechtsgrundlage angegeben wurde, also ist die Interpretation des Art. 52 als Ermächtigungsgrundlage für städtische Normsetzung anscheinend abweichend beurteilt worden - mit den Erlassen von 1877/78 hat die Hauptstadt nur einen bestimmten Aspekt der Einwirkung auf die Gewässer sicher geregelt.

Inwiefern lässt sich nun von einem kommunalen Gestaltungsspielraum bei der Normgebung sprechen, wenn zuvor die Staatsverwaltung mit Kreisregierung und Innenministerium einbezogen werden musste? Die größere, im zitierten Schreiben von 1867 bekräftigte Sachkenntnis bezüglich der lokalen Verhältnisse erschwerte es den höheren Behörden zumindest, ein vorgelegtes Normenwerk ohne triftige Gründe abzulehnen. Da die Initiative

84 Bekanntmachung: Anlage und Einrichtung der Abtritte, Dung- und Versitzgruben betr. (vom 5. Mai 1864), in: Münchener Amtsblatt, (1864), S. 580-584.

85 Ortspolizeiliche Vorschriften vom 3./16. November 1877, die Einleitung von Entwässerungen in den Schlachthauskanal betreffend, in: Münchener Gemeindezeitung, (1877), S. 1115-1118. Dazu auch: Stadtarchiv München, Sign. DE-1992-BAUA-TB-0742. 
vom Magistrat ausging, hätte eine Ablehnung des Erlasses unter Verweis auf eine fehlende Zuständigkeit auch zur Folge gehabt, dass Ressourcen höherer Behörden in die Rechtssetzung hätten investiert werden müssen. Ein solches städtisches Vorgehen benötigte aber die Mitwirkung der höheren Behörden. Anders als der Umgang mit Einzelfällen konnte der Erlass allgemeiner Vorschriften kaum der Aufmerksamkeit von Kreisregierung und Ministerium entgehen.

\section{RESÜMEE}

Das Wasserrecht war bis zur 2. Hälfte des 19. Jahrhunderts im Königreich Bayern lokal unterschiedlich geregelt und nicht mit Ausschließlichkeitsanspruch normiert; dies sollte nicht wertend als defizitär bezeichnet werden, da ein enger gesteckter Regelungsrahmen für die älteren Benutzungsbedürfnisse nicht erforderlich war. Mit der Verbreitung der Kodifikationsidee und der zunehmenden Industrialisierung kam es ab ca. $1850 \mathrm{zu}$ einer intensiven Verrechtlichung von Gewässerbenutzungen und anderen Aspekten des Wasserrechts. Das Wasserbenützungsgesetz von 1852 für das Königreich Bayern bedeutete für die Haupt- und Residenzstadt München nach den Reformen um 1800 eine weitere erhebliche Zurückdrängung kommunaler Gestaltungsspielräume. Trotz der faktisch größeren Dichte von Gewässereinwirkungen im urbanen Raum wurde auf normativer Ebene keine besondere Rücksicht auf engere Siedlungsverhältnisse genommen. Besonderes Münchner Wasserrecht gab es im monarchischen Verwaltungsstaats des 19. Jahrhunderts nur noch unter enger Kooperation mit den höheren Behörden sowie als ein vom Gesetzgeber anerkanntes Altrecht, wie dem schon 1724 bestätigten, 1852 weiterhin anerkannten und 1855 bekräftigten Burgfriedensprivileg von 1460. Beispiele für die überschaubaren verbleibenden Gestaltungsspielräume der Stadt München im Umgang mit Einzelfällen sind die Möglichkeit der Nichtinformation der übergeordneten Behörden über wasserrechtliche Gestattungen sowie die Umdeutung von Privatrechtsverhältnissen in Verwaltungshandeln auf der Basis des Wasserbenützungsgesetzes. Übergreifend lassen sich damit kooperative und nichtkooperative Formen städtischer Gestaltungsspielräume unterscheiden. 


\section{SWOBODA DECYDOWANIA MIAST O REGULACJI ZASOBÓW WODNYCH PRZED I PO WPROWADZENIU \\ BAWARSKICH USTAW O PRAWIE WODNYM Z 1852 ROKU}

\section{STRESZCZENIE}

W XIX wieku monarchia bawarska ograniczyła swobodę kształtowania przestrzeni przez władze miejskie. Na tym tle ustawa o gospodarowniu wodą z 1852 roku może być interpretowana jako silniejsza kontrola użytkowania zasobów wodnych przez władze centralne. Na przykładzie Monachium, miasta-stolicy oraz rezydencji władców, znajdującego się pod szczególną obserwacją państwa, można rozróżnić wiele form swobody decydowania o kształcie przestrzeni przez miasta.

Tłumaczenie Renata Skowrońska

\section{GESTALTUNGSSPIELRÄUME STÄDTISCHER REGULIERUNG VON GEWÄSSERN VOR UND NACH DEN BAYERISCHEN WASSERGESETZEN VON 1852}

\section{ZUSAMMENFASSUNG}

Die bayerische Monarchie beschränkte im Laufe des 19. Jahrhunderts die Gestaltungsspielräume städtischer Behörden. Vor diesem Hintergrund lässt sich das Wasserbenützungsgesetz von 1852 als stärkere zentrale Steuerung von Gewässerbenutzungen interpretieren. Am Beispiel der besonders unter staatlicher Beobachtung stehenden Haupt- und Residenzstadt München lassen sich mehrere Formen kommunaler Gestaltungsspielräume unterscheiden.

\section{THE FREEDOM OF CITIES TO DECIDE ON THE REGULATION OF WATER RESOURCES BEFORE AND AFTER THE INTRODUCTION OF THE BAVARIAN USAGE OF WATER LAW OF 1852}

\section{SUMMARY}

In the $19^{\text {th }}$ c., the Bavarian monarchy limited the freedom of the urban authorities to shape space. In view of this, the Bavarian Usage of Water Law of 1852 may be interpreted as a stronger control of central authorities over the use of water resources. On the example of Munich, the capital city which was under special observation by the rulers of the country, one can distinguish between many forms of city freedom to decide on the regulations of water resources.

Translated by Anna Maleszka 


\section{SŁOWA KLUCZOWE / SCHLAGWORTE / KEYWORDS}

- ustanawianie norm; bawarska ustawa o gospodarowaniu wodą; prawo wodne; prawa miejskie; praktyka administracyjna

- Normsetzung; Bayerisches Wasserbenützungsgesetz; Wasserrecht; Stadtrecht; Verwaltungspraxis

- setting standards; Bavarian Usage of Water Law; water law; urban laws; administrative practice

\section{BIBLIOGRAFIA / BIBLIOGRAFIE / BIBLIOGRAPHY}

\section{ŹróDłA ARCHIWALNE / ARCHIVALISCHE QUELLEN / ARCHIVAL SOURCES}

Stadtarchiv München: Sign. DE-1992-BAUA-TB-0493, DE-1992-BAUA-TB-0742, DE1992-BUG-005, DE-1992-BUG-007, DE-1992-BUG-571.

\section{ŹródłA DRUKOWANE / GEDRUCKTE QUELLEN / PRINTED SOURCES}

Bekanntmachung: Anlage und Einrichtung der Abtritte, Dung- und Versitzgruben betr. (vom 5. Mai 1864), in: Münchener Amtsblatt, (1864), S. 580-584.

Das bayerische Landrecht (Codex Maximilianeus Bavaricus civilis) vom Jahre 1756 in seiner heutigen Geltung, hg. v. M. Danzer, (1894).

Edikt über das Gemeinde-Wesen (vom 24. September 1808), in: Königlich-baierisches Regierungsblatt, (1808), Sp. 2405-2431.

Gesetz über die Benützung des Wassers (vom 28. Mai 1852), in: Gesetzblatt für das Königreich Baiern, (1851/1852), Sp. 489-542.

Gesetz, vom 1. July 1834, die Revision der Verordnung vom 17. May 1818, die Verfassung und Verwaltung der Gemeinden betreffend, in: Gesetzblatt für das Königreich Baiern, (1834), Sp. 109-132.

Instruktion der Polizei-Direktionen in den Städten (vom 24. September 1808), in: Königlich-Baierisches Regierungsblatt, (1808), Sp. 2509-2532.

Königliche Verordnung: das Verhältnis zwischen der Polizey-Direction und dem Magistrate der Haupt- und Residenz-Stadt München betreffend (vom 15. September 1818), in: Gesetzblatt für das Königreich Baiern, (1818), Sp. 573-616.

Königliche Verordnung: die künftige Verfassung und Verwaltung der Gemeinden im Königreiche betreffend (vom 17. Mai 1818), in: Gesetzblatt für das Königreich Baiern, (1818), Sp. 49-96.

Königliche Verordnung: die künftige Verfassung und Verwaltung der Gemeinden im Königreiche betreffend (vom 17. Mai 1818), in: Die bayerischen Gemeindeordnungen 1808-1945, hg. v. F.-L. Knemeyer, (Schriften zur öffentlichen Verwaltung 41), (1994), S. 32-62.

Landrecht, Policey- Gerichts- Malefitz- vnd andere Ordnungen. Der Fürstenthumben Obern vnd Nidern Bayrn, (1616).

Verfassungsurkunde des Königreich Baierns (vom 26. Mai 1818), in: Gesetzblatt für das Königreich Baiern, (1818), Sp. 101-140. 


\section{LITERATURA / LITERATUR / LITERATURE}

Bauer R., Stadt und Stadtverfassung im Umbruch. Niedergang, Ende und Neubegründung kommunaler Eigenständigkeit 1767 bis 1818, in: Geschichte der Stadt München, hg. v. dems., (1992), S. 244-273.

Behrens C., Die Wassergesetzgebung im Herzogtum Braunschweig nach Bauernbefreiung und industrieller Revolution. Zur Genese des Wasserrechts im bürgerlichen Rechtsstaat, (Rechtsgeschichtliche Studien 30, 2009).

Collin P., Einleitung: „Gesellschaftliche Selbstregulierung“ und „Regulierte Selbstregulierung“ - ertragreiche Analysekategorien für eine (rechts-)historische Perspektive?, in: Selbstregulierung im 19. Jahrhundert. Zwischen Autonomie und staatlichen Steuerungsansprüchen, hg. v. dems et al., (Schriften zur europäischen Rechtsgeschichte 259, Moderne Regulierungsregime 1, 2011), S. 3-31.

Collin P., Kommunalrecht unter Regulierungsdruck in der Weimarer Zeit, in: Regulierte Selbstregulierung im frühen Interventions- und Sozialstaat, hg. v. dems et al., (Schriften zur europäischen Rechtsgeschichte 270, Moderne Regulierungsregime 2, 2012), S. 145-163.

Fisch S., Stadtplanung im 19. Jahrhundert. Das Beispiel München bis zur Ära Theodor Fischer, (1988).

Franz M. R., Rechtliche Bestimmungen zum Wasserbau im Herzog- und Kurfürstentum Bayern, in: Altbayerische Flußlandschaften an Donau, Lech, Isar und Inn: handgezeichnete Karten des 16. bis 18. Jahrhunderts aus dem Bayerischen Hauptstaatsarchiv, (1998), S. 290-296.

Geffcken H., Zur Geschichte des deutschen Wasserrechts, in: Zeitschrift der Savigny-Stiftung. Germanistische Abteilung, 21 (1900), S. 173-217.

Gömmel R., Gewerbe, Handel und Verkehr, in: Handbuch der bayerischen Geschichte, 4: Das neue Bayern, von 1800 bis zur Gegenwart, 2: Die innere und kulturelle Entwicklung, (2007), S. 216-299.

Held-Daab U., Das freie Ermessen: von den vorkonstitutionellen Wurzeln zur positivistischen Auflösung der Ermessenslehre, (Schriften zum öffentlichen Recht 706, 1996).

Heydenreuther R., Die Isar in der Rechtsgeschichte. Anmerkungen zur Entwicklung des bayerischen Wasserrechts, in: Die Isar: ein Lebenslauf. Ausstellung im Münchner Stadtmuseum vom 5. Mai bis 25. September 1983, hg. v. M.-L. Plessen, (1983), S. 52-63.

Kohl W., Recht und Geschichte der alten Münchner Mühlen, (Miscellanea Bavarica Monacensia 15. Neue Schriftenreihe des Stadtarchivs München 32, 1969).

Lück H., Mühle, Mühlenrecht, in: Handwörterbuch zur Deutschen Rechtsgeschichte, 3 ( $\left.{ }^{2} 2016\right)$, Sp. 447-448.

Meisner C., Das in Bayern geltende Nachbarrecht: mit Berücksichtigung des Wasserrechts, (1901 und $\left.{ }^{2} 1910\right)$.

Münch P., Stadthygiene im 19. und 20. Jahrhundert: die Wasserversorgung, Abwasser- und Abfallbeseitigung unter besonderer Berücksichtigung Münchens, (Schriftenreihe der Historischen Kommission bei der Bayerischen Akademie der Wissenschaften 49, 1993).

Pözl J., Die bayerischen Wassergesetze vom 28. Mai 1852. Mit einem Anhange, die Ordnung der Schiff- und Floßfahrt auf den bayerischen Flüssen, Seen und Kanälen betreffend, (1862).

Rädlinger C., Geschichte der Münchner Stadtbäche, (2004). 
Reuß H., Die bayerischen Wassergesetze vom 28. Mai 1852: mit Ergänzungsgesetzen, Vollzugserlassen, Motiven, oberstrichterlichen Erkenntnissen und Erläuterungen, (Die bayerischen Kulturgesetze nebst Vollzugsvorschriften 3, 1890).

Seiler H., Die Gewässerbenutzungen und ihre Rechtsgrundlagen im Verlauf der Geschichte des Wasserrechts. Ein vergleichender Überblick, (1976).

Seydel M., Bayerisches Staatsrecht, 5 (1891).

Verwaltungsverfahrensgesetz, hg. v. P. Stelkens, H. J. Bonk, M. Sachs, (2018).

Volkert W., Innere Verwaltung, in: Handbuch der bayerischen Ämter, Gemeinden und Gerichte 1799-1980, hg. v. dems., (1983).

Weis E., Die Begründung des modernen bayerischen Staates unter König Max I. (1799-1825), in: Handbuch der bayerischen Geschichte, 4: Das neue Bayern, von 1800 bis zur Gegenwart, 1: Staat und Politik, (2003), S. 3-126.

Zerback R., Unter der Kuratel des Staates - Die Stadt zwischen dem Gemeineedikt von 1818 und der Gemeindeordnung von 1869, in: Geschichte der Stadt München, hg. v. R. Bauer, (1992), S. 244-273. 